\title{
Prognostic Impact of Sarcopenia in Patients With Biliary Tract Cancer Undergoing Chemotherapy
}

\author{
KOKI MEGURO ${ }^{1}$, KUNIHIRO HOSONO $^{2}$, MOTOKAZU SATO ${ }^{1}$, YUICHI SUGIMOTO ${ }^{1}$, YUSUKE TAKAI ${ }^{1}$, \\ YUSUKE KURITA $^{2}$, KENJI KANOSHIMA ${ }^{1}$, TOMOKI SHIMIZU ${ }^{1}$, EIJI SAKAI ${ }^{1}$ and ATSUSHI NAKAJIMA ${ }^{2}$ \\ ${ }^{1}$ Gastroenterology Division, Yokohama Sakae Kyosai Hospital, Yokohama, Japan; \\ ${ }^{2}$ Department of Gastroenterology and Hepatology, Yokohama City University School of Medicine, Yokohama, Japan
}

\begin{abstract}
Aim: Sarcopenia affects the treatment of various cancer types but its impact on chemotherapy efficacy and prognosis in biliary tract cancer remains unclear. Thus, we evaluated whether sarcopenia independently affects the outcome of chemotherapy for biliary tract cancer. Patients and Methods: Data of 50 patients who underwent chemotherapy for biliary tract cancer at two affiliated centres were retrospectively analysed. The association of clinical factors, including sarcopenia, with overall survival and time to treatment failure was analysed. Results: Sarcopenia was an independent factor negatively influencing overall survival and time to treatment failure in univariate and multivariate analyses (median overall survival, sarcopenic vs. non-sarcopenic patients: 10.6 vs. 16.6 months; hazard ratio $=2.19, p=0.018$; time to treatment failure: 5.3 vs. 13.1 months, hazard ratio=2.50, $p=0.019$ ). Conclusion: Sarcopenia may affect the efficacy of chemotherapy and prognosis in biliary tract cancer. Thus, improving sarcopenia may improve the prognosis of patients with biliary tract cancer undergoing chemotherapy.
\end{abstract}

Chemotherapy is the last treatment option for patients with biliary tract cancer (1). Patient characteristics, such as age and the Eastern Cooperative Oncology Group performance status (ECOG PS) prior to chemotherapy, have been reported to influence the efficacy of chemotherapy for various carcinomas (2). Sarcopenia, a concept proposed by Rosenberg in the late 1980s (3), is defined as a decrease in skeletal muscle mass and muscle function, and it has been

This article is freely accessible online.

Correspondence to: Kunihiro Hosono, MD, Ph.D., Gastroenterology Division, Yokohama City University School of Medicine, 3-9 Fukuura, Kanazawa-ku, Yokohama, 236-0004 Japan. Tel: +81 457872640, Fax: +81457843546, e-mail: hiro1017@yokohama-cu.ac.jp

Key Words: Biliary tract cancer, chemotherapy, sarcopenia. used as an indicator of activities of daily living and nutritional status (4). Sarcopenia may affect the prognosis of various cancer types including lung, gastric, colorectal, and pancreatic, by diminishing the effect of chemotherapy $(5,6)$. The association between sarcopenia and efficacy of chemotherapy in biliary tract cancer has not yet been fully investigated and remains unknown. Hence, this study aimed to evaluate whether sarcopenia independently affects the efficacy of chemotherapy for biliary tract cancer.

\section{Patients and Methods}

Patients. Using our electronic health records databases, we retrospectively collected and studied the data of patients with biliary tract cancer who underwent chemotherapy at Yokohama Sakae Kyosai Hospital and Yokohama City University Hospital between November 2012 and February 2019. The diagnosis of most biliary tract cancers was based on histological findings obtained by endoscopic retrograde cholangiopancreatography. Where histological determination was not possible, such as in patients with gallbladder cancer, diagnosis was based on imaging findings and tumour markers.

Here, we only included patients with ECOG PS0-1, due to the poorer prognosis of patients with PS2 or more which might have influenced our results (2). In addition, given that the tumour stage affects prognosis, we included only those with stage III and IV tumours which are associated with a 5-year survival rate of approximately $30 \%$ or less in Japan, and excluded patients with tumours of lower stages (7). Tumour staging was determined based on the eighth edition of the Union for International Cancer Control Tumour Node and Metastasis Classification of Malignant Tumours (8). Other exclusion criteria included patients without sufficient follow-up or clinical data, those with severe comorbidities that may affect prognosis, and those whose cause of death was not likely to be related to biliary tract cancer (for example, pneumonia, heart failure, or myocardial infarction). All patients provided their written informed consent prior to starting chemotherapy.

Data collection. We collected clinical data, including, age, sex, ECOG PS, laboratory data (complete blood count and aspartate aminotransferase, alanine aminotransferase, total bilirubin, alkaline phosphatase, $\gamma$-glutamyl transpeptidase, albumin, and C-reactive protein), and levels of serum tumour markers, such as carcinoembryonic antigen and carbohydrate antigen 19-9 (CA19-9). 
Each cut-off value was determined by referring to previous reports (9-11). Additionally, we also analysed comorbidities (hypertension, dyslipidaemia, type 2 diabetes, and cholelithiasis), body mass index, tumour site (bile duct or gallbladder), location of metastasis (lung and liver), and chemotherapy regimen. These values were measured within 4 weeks from the start of chemotherapy. This study was approved by the Research Ethics Committee of Yokohama Sakae Kyosai Hospital (20181217-4) and Yokohama City University Certified Institutional Review Board (B201000005).

Treatment strategy. In Japan, a combination therapy with gemcitabine plus cisplatin (GC therapy), gemcitabine plus S-1 (GS therapy), and gemcitabine or S-1 monotherapy are approved for biliary tract cancer treatment (1). The chemotherapy regimen was determined according to third edition of the Clinical Practice Guidelines for the Management of Biliary Tract Cancers, published by the Japanese Society of Hepato-Biliary-Pancreatic Surgery (1). Generally, either GC or GS therapy was selected as a first-line regimen. Depending on the patient's age, general condition, comorbidities, and patient preference, single-agent gemcitabine or S-1 therapy was occasionally chosen as an optional first-line treatment. Furthermore, for each regimen, dose modification was based on the occurrence of adverse effects and the patient's status. These choices were made at the discretion of the attending physician and with the patient's consent. The efficacy of chemotherapy was evaluated by computed tomographic imaging. When a patient was diagnosed with progressive disease as defined by the revised Response Evaluation Criteria in Solid Tumours guideline version 1.1 (12), they were administered a second-line regimen or the treatment was discontinued, depending on the physician's assessment based on a consideration of various factors.

Sarcopenia. The presence of sarcopenia was assessed using Ziostation2 (Ziosoft, Tokyo, Japan) and Vincent (Fuji film, Tokyo, Japan) by measuring the area of the psoas muscle at the third lumbar spine on computed tomographic images taken within 4 weeks before chemotherapy initiation. Simultaneously, subcutaneous fat and visceral fat were also measured. Each tissue HU threshold was set at -29 to $150 \mathrm{HU}$ for the iliopsoas muscle and -150 to $-30 \mathrm{HU}$ for the adipose tissue, as per previous reports $(9,13)$.

The cross-sectional area of the psoas muscle was normalized for the patient height by calculating the psoas muscle index (PMI: $\mathrm{cm}^{2} / \mathrm{m}^{2}$ ). To define sarcopenia, we used the sex-specific PMI cutoff values provided by the Japan Society of Hepatology as follows: PMI $<3.92 \mathrm{~cm}^{2} / \mathrm{m}^{2}$ for women and $<6.36 \mathrm{~cm}^{2} / \mathrm{m}^{2}$ for men (14). We also calculated the subcutaneous adipose tissue index, visceral adipose tissue index (VATI), and total adipose tissue index (ATI) of the third lumbar vertebra. Similarly, each adipose tissue area was normalized for height (15). Cut-off values for subcutaneous adipose tissue index, VATI, and ATI were defined as previously reported (9).

Statistical analyses. The study endpoint was overall survival (OS), which was defined as the period from the start of the first chemotherapy until death. Additionally, we also investigated prognostic factors for the time to treatment failure (TTF), which was defined as the period from chemotherapy initiation to discontinuation of treatment for any reason, including the appearance of adverse effects, disease progression, or patient complaint or death.

In the analysis of the relationship between sarcopenia and individual factors, the Mann-Whitney $U$-test was applied for continuous variables, and Fisher's exact test or Pearson's chisquared test was applied for categorical data. The OS and TTF analyses were evaluated using the Kaplan-Meier method. Differences between each group were assessed using the log-rank test for univariate analyses, and the Cox proportional hazard model for multivariate analyses. Tests were two-sided, with the significance level set at $p<0.05$. All statistical analyses were performed using EZR software (16).

\section{Results}

The data of 62 eligible patients were evaluated from the database. Twelve patients were excluded after applying the exclusion criteria. Hence, 50 patients were finally included in this retrospective analysis. Patient characteristics are summarized in Table I. The median age of patients was 75 (range $=48-87$ ) years, and they were predominantly male $(60 \%)$. There were 31 patients $(62 \%)$ with cholangiocarcinoma (distal cholangiocarcinoma plus hilar cholangiocarcinoma) and $19(38 \%)$ with gallbladder cancer. Initially, 24 patients $(48 \%)$ had metastases, of whom 14 (28\%) had liver metastases, 10 (20\%) had lung metastases, and five (10\%) had other metastases, such as peritoneal seeding. There were 22 patients with stage III and 28 with stage IV. Regarding the chemotherapy regimen, 41 patients $(82 \%)$ received a regimen containing gemcitabine, and nine patients $(18 \%)$ received a regimen without gemcitabine (S-1). Furthermore, 25 patients (50\%) received second-line chemotherapy or later lines.

In this survey, the average PMI was $5.24 \mathrm{~cm}^{2} / \mathrm{m}^{2}(5.82$ $\mathrm{cm} / \mathrm{m}^{2}$ for men and $4.35 \mathrm{~cm}^{2} / \mathrm{m}^{2}$ for women). Consequently, $28(56.0 \%)$ patients were classified as having sarcopenia. There were no significant differences in clinical background factors, such as the age, sex, tumour staging or presence of metastasis between the sarcopenic and non-sarcopenic groups (Table I).

The results of the univariate and multivariate analyses of the factors for the OS are shown in Table II. In the univariate analysis, sarcopenia, lung metastasis, liver metastasis, gall bladder tumour, and high CA19-9 level were identified as significant factors negatively affecting OS $(p<0.05)$. A multivariate analysis was then performed, and the results showed that sarcopenia [hazard ratio $(\mathrm{HR})=2.19,95 \%$ confidence interval $(\mathrm{CI})=1.14-4.23 ; p=0.018$ and CA19-9 $(\mathrm{HR}=4.62,95 \% \mathrm{CI}=2.07-10.31 ; p<0.001)]$ were significant independent factors.

Table III shows the results of univariate and multivariate analyses of factors predicting the TTF. Univariate analysis showed that sarcopenia, ECOG PS, CA19-9, VATI, and ATI may be associated with TTF $(p<0.05)$. Furthermore, multivariate analysis demonstrated that sarcopenia $(H R=2.50$, 95\% CI=1.15-5.43; $p=0.019$ ) was the only independent factor. Hence, sarcopenia was a significant factor for both OS and TTF.

There were 28 patients with sarcopenia and 22 without. Kaplan-Meier curves for OS and TTF for patients with and 
Table I. Patient characteristics and comparisons between patients with sarcopenia and without sarcopenia.

\begin{tabular}{|c|c|c|c|c|c|}
\hline Characteristic & & Total $(\mathrm{n}=50)$ & Sarcopenia $(\mathrm{n}=28,56 \%)$ & Non-sarcopenia $(\mathrm{n}=22,44 \%)$ & $p$-Value \\
\hline Age, (range) & Median (range) & $75(48-87)$ & $73(48-83)$ & $77(70-87)$ & 0.017 \\
\hline Gender, n (\%) & Male & $30(60)$ & $19(38)$ & $11(22)$ & 0.25 \\
\hline \multirow[t]{2}{*}{ ECOG PS, n (\%) } & 0 & $27(54)$ & $13(26)$ & $14(28)$ & 0.38 \\
\hline & 1 & $23(46)$ & $15(30)$ & $8(16)$ & \\
\hline \multirow[t]{2}{*}{ Site of tumor, n (\%) } & Bile duct $(\mathrm{Bp}+\mathrm{Bd})$ & $31(62)$ & $14(28)$ & $17(34)$ & 0.055 \\
\hline & Gall bladder & $19(38)$ & $14(28)$ & $5(10)$ & \\
\hline \multirow[t]{3}{*}{ Metastasis, n (\%) } & Liver & $14(28)$ & $8(16)$ & $6(12)$ & $>0.99$ \\
\hline & Lung & $10(20)$ & $7(14)$ & $3(6)$ & 0.48 \\
\hline & Peritoneum & $4(8)$ & $1(2)$ & $3(6)$ & 0.30 \\
\hline Recurrent, n (\%) & Yes & $12(24)$ & $7(14)$ & $5(10)$ & $>0.99$ \\
\hline \multirow[t]{2}{*}{ Stage, $n(\%)$} & III & $26(52)$ & $11(22)$ & $11(22)$ & 0.568 \\
\hline & IV & $28(56)$ & $17(34)$ & $11(22)$ & \\
\hline \multirow[t]{4}{*}{ First line, n (\%) } & GC & $17(34)$ & $10(20)$ & $7(14)$ & 0.407 \\
\hline & GS & $8(16)$ & $6(12)$ & $2(4)$ & \\
\hline & Gemcitabine & $16(32)$ & $9(18)$ & $7(14)$ & \\
\hline & S-1 & $9(18)$ & $3(6)$ & $6(12)$ & \\
\hline \multirow[t]{2}{*}{ Second line, n (\%) } & Yes & $25(50)$ & $13(26)$ & $12(24)$ & 0.78 \\
\hline & Diabetes & $15(30)$ & $9(18)$ & $6(12)$ & 0.77 \\
\hline \multirow[t]{3}{*}{ Comorbidity, n, n (\%) } & Hypertension & $29(58)$ & $17(34)$ & $12(24)$ & 0.78 \\
\hline & Lipidemia & $17(34)$ & $8(16)$ & $9(18)$ & 0.39 \\
\hline & Gall stone & $11(22)$ & $6(12)$ & $5(10)$ & $>0.99$ \\
\hline $\mathrm{WBC}, \mathrm{n} / \mu \mathrm{l}$ & Median (IQR) & $5,800(4,600-6,800)$ & $5,750(4,750-6,550)$ & $6,250(4,525-7,100)$ & 0.61 \\
\hline Albumin, $\mathrm{g} / \mathrm{dl}$ & Median (IQR) & $3.52(3.1-3.94)$ & $3.66(3.10-3.96)$ & $3.44(3.14-3.89)$ & 0.63 \\
\hline $\mathrm{CRP}, \mathrm{mg} / \mathrm{dl}$ & Median (IQR) & $0.45(0.21-1.05)$ & $0.30(0.18-0.81)$ & $0.68(0.30-1.41)$ & 0.12 \\
\hline AST, IU/1 & Median (IQR) & $28(21-48)$ & $29(23-44)$ & $24(20-48)$ & 0.57 \\
\hline ALT, IU/1 & Median (IQR) & $26(18-48)$ & $29(22-54)$ & $21(15-38)$ & 0.11 \\
\hline $\mathrm{T}-\mathrm{Bil}, \mathrm{mg} / \mathrm{dl}$ & Median (IQR) & $0.70(0.60-1.0)$ & $0.7(0.6-0.9)$ & $0.8(0.6-1.0)$ & 0.64 \\
\hline ALP, IU/1 & Median (IQR) & $408(280-713)$ & $385(311-844)$ & $440(243-475)$ & 0.67 \\
\hline$\gamma$-GTP, IU/1 & Median (IQR) & $100(39-279)$ & $92(39-313)$ & $106(47-207)$ & 0.79 \\
\hline CA19-9, U/ml & Median (IQR) & $315(39-1,196)$ & $616(248-1,223)$ & $130(27-1,059)$ & 0.17 \\
\hline $\mathrm{CEA}, \mathrm{ng} / \mathrm{ml}$ & Median (IQR) & $4.30(2.70-7.60)$ & $4.2(3.1-8.6)$ & $4.8(2.4-7.5)$ & 0.49 \\
\hline BMI, $\mathrm{kg} / \mathrm{m}^{2}$ & Median (IQR) & $21.6(19.6-23.7)$ & $21.9(18.9-23.6)$ & $21.3(20.1-23.4)$ & 0.85 \\
\hline SATI, $\mathrm{cm}^{2} / \mathrm{m}^{2}$ & Median (IQR) & $30.6(19.2-38.7)$ & $28.2(16.6-38.9)$ & $35.6(20.2-38.6)$ & 0.31 \\
\hline VATI, $\mathrm{cm}^{2} / \mathrm{m}^{2}$ & Median (IQR) & $33.9(21.2-57.7)$ & $44.3(22.9-58.1)$ & $31.1(18.0-53.0)$ & 0.45 \\
\hline ATI, $\mathrm{cm}^{2} / \mathrm{m}^{2}$ & Median (IQR) & $63.2(45.4-95.1)$ & $78.1(42.3-95.0)$ & $60.0(48.9-94.3)$ & 0.75 \\
\hline
\end{tabular}

ALP: Alkaline phosphatase; ALT: alanine aminotransferase; AST: aspartate aminotransferase; ATI: adipose tissue index; Bd: distal bile duct; BMI: body mass index; Bp: perihilar bile duct; CA19-9: carbohydrate antigen 19-9; CEA: carcinoembryonic antigen; CRP: C-reactive protein; ECOG PS: Eastern Cooperative Oncology Group performance status; GC: gemcitabine plus cisplatin; GEM: gemcitabine; GS: gemcitabine plus S-1; IQR: interquartile range; SATI: subcutaneous adipose tissue index; T-Bil: total bilirubin; VATI: visceral adipose tissue index; WBC: white blood cell; $\gamma \mathrm{GTP}: \gamma$-glutamyl transpeptidase. Statistically significant $p$-values are shown in bold.

without sarcopenia are shown in Figure 1. The median OS for the entire cohort was 12.6 months. The median OS of patients with sarcopenia was shorter than that of patients without sarcopenia (10.6 vs. 16.6 months, $p=0.015$ ) (Figure 1A). The median TTF for patients overall was 8.5 months (Figure 1B). Similarly, the TTF in patients with sarcopenia was shorter than in those without (5.3 vs. 13.1 months, respectively, $p=0.045$ ).

\section{Discussion}

This study evaluated the association of sarcopenia with the efficacy of chemotherapy in patients with biliary tract cancer. Our results showed that sarcopenia is an independent prognostic factor for patients with biliary tract cancer treated with chemotherapy. Previous studies have reported that sarcopenia affects the efficacy of chemotherapy in other carcinoma types $(9,17)$; our results show that sarcopenia affects chemotherapy in patients with biliary tract cancer.

It has been noted that sarcopenia is associated with prognosis and treatment response of various carcinomas. Okumura et al. evaluated the association between surgical treatment and sarcopenia and found that the low-PMI (sarcopenia) group had significantly poorer OS than the normal-PMI (non-sarcopenia) group in patients who had undergone resection of biliary tract cancer, except for those with intrahepatic cholangiocarcinoma. They found that the 
Table II. Univariate and multivariate analyses for overall survival.

\begin{tabular}{|c|c|c|c|c|c|c|c|}
\hline \multirow[b]{2}{*}{ Factor } & \multirow[b]{2}{*}{ Subgroup } & \multirow[b]{2}{*}{ OS, months (median) } & \multicolumn{2}{|c|}{ Univariate } & \multicolumn{3}{|c|}{ Multivariate } \\
\hline & & & $95 \% \mathrm{CI}$ & $p$-Value & HR & $95 \% \mathrm{CI}$ & $p$-Value \\
\hline \multirow[t]{2}{*}{ Age } & $<75$ Years & 11.0 & $6.9-14.2$ & 0.29 & & & \\
\hline & $\geq 75$ Years & 13.7 & $11.4-21.5$ & & & & \\
\hline \multirow[t]{2}{*}{ Gender } & Female & 11.8 & 6.6-17.4 & 0.91 & & & \\
\hline & Male & 12.8 & $8.2-16.4$ & & & & \\
\hline \multirow[t]{2}{*}{ ECOG PS } & 1 & 8.5 & $5.6-12.8$ & 0.058 & & & \\
\hline & 0 & 14.5 & $11.2-19.7$ & & & & \\
\hline \multirow[t]{2}{*}{ Tumor site } & Bile duct & 14.5 & $8.5-19.0$ & 0.034 & 1.89 & $1.01-3.95$ & 0.056 \\
\hline & Gall bladder & 11.2 & $6.9-12.8$ & & 1 & & \\
\hline \multirow[t]{2}{*}{ Liver metastasis } & Present & 10.0 & $5.7-14.2$ & 0.031 & 1.19 & $0.57-2.51$ & 0.64 \\
\hline & Absent & 13.1 & $9.8-19.0$ & & 1 & & \\
\hline \multirow[t]{2}{*}{ Lung metastasis } & Present & 7.7 & $0.8-14.5$ & 0.049 & 1.42 & $0.59-3.41$ & 0.43 \\
\hline & Absent & 12.8 & $11.2-16.6$ & & 1 & & \\
\hline \multirow[t]{2}{*}{ Recurrent } & Present & 12.9 & $2-22$ & 0.66 & & & \\
\hline & Absent & 12.6 & $9.5-14.6$ & & & & \\
\hline \multirow[t]{2}{*}{ First line } & Included GEM & 11.4 & $8.2-14.5$ & 0.38 & & & \\
\hline & S-1 & 16.6 & $5.3-25.6$ & & & & \\
\hline \multirow[t]{2}{*}{ Second line } & Absent & 12.8 & $6.5-16.6$ & 0.52 & & & \\
\hline & Present & 12.1 & $8.5-16.4$ & & & & \\
\hline \multirow{2}{*}{ Diabetes } & Present & 7.7 & $5.3-12.4$ & 0.21 & & & \\
\hline & Absent & 14.3 & $11.4-16.7$ & & & & \\
\hline \multirow[t]{2}{*}{ Hypertension } & Present & 11.2 & $6.8-14.6$ & 0.16 & & & \\
\hline & Absent & 14.5 & $8.5-17.4$ & & & & \\
\hline Lipidemia & Present & 13.1 & $9.5-19.7$ & 0.38 & & & \\
\hline & Absent & 12.1 & $6.8-14.5$ & & & & \\
\hline Gallstone & Present & 12.8 & $5.3-17.4$ & 0.98 & & & \\
\hline & Absent & 12.4 & $8.2-16.5$ & & & & \\
\hline WBC & $\geq 8000 / \mu \mathrm{l}$ & 9.8 & $5.7-14.2$ & 0.10 & & & \\
\hline & $<8000 / \mu 1$ & 12.8 & 10.7-16.6 & & & & \\
\hline AST & $\geq 27$ IU/1 & 12.8 & $10.7-16.4$ & 0.44 & & & \\
\hline & $<27$ IU/1 & 12.2 & $6.5-16.6$ & & & & \\
\hline ALT & $\geq 33 \mathrm{IU} / 1$ & 10.4 & $6.5-16.7$ & 0.51 & & & \\
\hline & $<33 \mathrm{IU} / 1$ & 12.8 & $10.7-16.4$ & & & & \\
\hline T-Bil & $\geq 1.2 \mathrm{IU} / 1$ & 17.0 & 4.3-19.7 & 0.68 & & & \\
\hline & $<1.2 \mathrm{IU} / 1$ & 12.4 & $9.5-14.5$ & & & & \\
\hline ALP & $\geq 334 \mathrm{IU} / 1$ & 12.8 & $6.6-17.4$ & 0.62 & & & \\
\hline & $<334 \mathrm{IU} / 1$ & 12.1 & $11.2-14.5$ & & & & \\
\hline$\gamma$-GTP & $\geq 66 \mathrm{IU} / 1$ & 12.3 & $6.8-16.7$ & 0.47 & & & \\
\hline & $<66$ IU/1 & 12.8 & $10.7-16.4$ & & & & \\
\hline Alb & $\geq 3.5 \mathrm{mg} / \mathrm{dl}$ & 14.2 & $12.1-16.7$ & 0.63 & & & \\
\hline & $<3.5 \mathrm{mg} / \mathrm{dl}$ & 11.0 & $5.6-14.6$ & & & & \\
\hline CRP & $\geq 1.0 \mathrm{mg} / \mathrm{dl}$ & 9.8 & $5.7-14.3$ & 0.12 & & & \\
\hline & $<1.0 \mathrm{mg} / \mathrm{dl}$ & 13.1 & $10.7-16.7$ & & & & \\
\hline CEA & $\geq 5.0 \mathrm{ng} / \mathrm{ml}$ & 10.6 & $10.7-16.6$ & 0.56 & & & \\
\hline & $<5.0 \mathrm{ng} / \mathrm{ml}$ & 14.4 & $6.9-19.0$ & & & & \\
\hline CA19-9 & $\geq 1,000 \mathrm{U} / \mathrm{ml}$ & 6.8 & $4.3-14.2$ & $<0.001$ & 4.62 & $2.07-10.31$ & $<0.001$ \\
\hline & $<1,000 \mathrm{U} / \mathrm{ml}$ & 14.6 & $12.1-19.0$ & & 1 & & \\
\hline BMI & $<22 \mathrm{~kg} / \mathrm{m}^{2}$ & 12.8 & $10.7-17.4$ & 0.41 & & & \\
\hline & $\geq 22 \mathrm{~kg} / \mathrm{m}^{2}$ & 12.1 & $6.8-16.4$ & & & & \\
\hline Sarcopenia & Present & 10.6 & $6.5-12.8$ & 0.015 & 2.19 & $1.14-4.23$ & 0.018 \\
\hline & Absent & 16.5 & $11.2-22.8$ & & 1 & & \\
\hline SATI & High & 12.8 & $10.7-19.7$ & 0.21 & & & \\
\hline & Low & 12.3 & $6.8-14.5$ & & & & \\
\hline VATI & High & 14.3 & $10.7-21.5$ & 0.078 & & & \\
\hline & Low & 11.0 & $6.8-14.6$ & & & & \\
\hline ATI & High & 16.7 & $10.7-21.5$ & 0.063 & & & \\
\hline & Low & 12.1 & 6.9-13.1 & & & & \\
\hline
\end{tabular}

ALP: Alkaline phosphatase; ALT: alanine aminotransferase; AST: aspartate aminotransferase; ATI: adipose tissue index; BMI: body mass index; Bp: perihilar bile duct; CA19-9: carbohydrate antigen 19-9; CEA: carcinoembryonic antigen; CRP: C-reactive protein; CI: confidence intervaI; ECOG PS: Eastern Cooperative Oncology Group performance status; GEM: gemcitabine; HR: hazard ratio; SATI: subcutaneous adipose tissue index; T-Bil: total bilirubin; VATI: visceral adipose tissue index; WBC: white blood cell; $\gamma$ GTP: $\gamma$-glutamyl transpeptidase. Statistically significant $p$-values are shown in bold. 
Table III. Univariate and multivariate analyses for time to treatment failure.

\begin{tabular}{|c|c|c|c|c|c|c|c|}
\hline \multirow[b]{2}{*}{ Factor } & \multirow[b]{2}{*}{ Subgroup } & \multirow[b]{2}{*}{ TTF, months (median) } & \multicolumn{2}{|c|}{ Univariate } & \multicolumn{3}{|c|}{ Multivariate } \\
\hline & & & $95 \% \mathrm{CI}$ & $p$-Value & HR & $95 \% \mathrm{CI}$ & $p$-Value \\
\hline \multirow[t]{2}{*}{ Age } & $<75$ Years & 6.8 & $4.8-11.3$ & 0.61 & & & \\
\hline & $\geq 75$ Years & 12.5 & $3.7-15.7$ & & & & \\
\hline \multirow[t]{2}{*}{ Gender } & Female & 7.6 & $3.3-14.4$ & 0.45 & & & \\
\hline & Male & 9.4 & $5.3-13.1$ & & & & \\
\hline \multirow[t]{2}{*}{ ECOG PS } & 1 & 5.0 & $3.0-8.3$ & 0.017 & 1.23 & $0.60-2.51$ & 0.57 \\
\hline & 0 & 13.1 & $6.4-15.8$ & & 1 & & \\
\hline \multirow[t]{2}{*}{ Site of tumor } & Bile duct & 12.8 & $4.4-14.4$ & 0.22 & & & \\
\hline & Gall bladder & 6.4 & $4.8-10.2$ & & & & \\
\hline \multirow[t]{2}{*}{ Liver metastasis } & Present & 7.6 & $3.3-13.1$ & 0.33 & & & \\
\hline & Absent & 9.4 & $4.8-13.6$ & & & & \\
\hline \multirow[t]{2}{*}{ Lung metastasis } & Present & 6.8 & $0.4-13.0$ & 0.12 & & & \\
\hline & Absent & 10.1 & $5.3-13.6$ & & & & \\
\hline \multirow[t]{2}{*}{ Recurrent } & Present & 11.1 & $1.3-17.7$ & 0.73 & & & \\
\hline & Absent & 7.6 & $5.3-12.8$ & & & & \\
\hline \multirow[t]{2}{*}{ First line } & Included GEM & 6.8 & $4.8-13.0$ & 0.58 & & & \\
\hline & S-1 & 11.3 & $2.1-17.7$ & & & & \\
\hline \multirow[t]{2}{*}{ Second line } & Absent & 5.3 & $3.0-13.6$ & 0.13 & & & \\
\hline & Present & 10.1 & $6.3-13.6$ & & & & \\
\hline \multirow{2}{*}{ Diabetes } & Present & 4.4 & $3.3-10.1$ & 0.14 & & & \\
\hline & Absent & 11.3 & $6.4-14.4$ & & & & \\
\hline \multirow[t]{2}{*}{ Hypertension } & Present & 6.4 & $4.5-12.8$ & 0.21 & & & \\
\hline & Absent & 10.1 & $4.4-15.8$ & & & & \\
\hline Lipidemia & Present & 12.2 & $4.8-15.8$ & 0.45 & & & \\
\hline & Absent & 6.8 & $4.4-12.8$ & & & & \\
\hline Gall stone & Present & 6.8 & $2.3-12.2$ & 0.64 & & & \\
\hline & Absent & 8.6 & $4.8-13.6$ & & & & \\
\hline WBC & $\geq 8,000 / \mu 1$ & 6.3 & $4.5-13.0$ & 0.19 & & & \\
\hline & $<8,000 / \mu 1$ & 10.1 & $5.3-13.6$ & & & & \\
\hline AST & $\geq 27 \mathrm{IU} / 1$ & 7.6 & $3.3-13.6$ & 0.50 & & & \\
\hline & $<27$ IU/1 & 10.8 & $5.3-13.1$ & & & & \\
\hline ALT & $\geq 33 \mathrm{IU} / 1$ & 7.6 & $3.3-14.4$ & 0.76 & & & \\
\hline & $<33 \mathrm{IU} / 1$ & 10.2 & $5.3-13.1$ & & & & \\
\hline T-Bil & $\geq 1.2 \mathrm{IU} / 1$ & 14.6 & $2.6-16.4$ & 0.53 & & & \\
\hline & $<1.2 \mathrm{IU} / 1$ & 7.5 & $5.3-12.2$ & & & & \\
\hline ALP & $\geq 334 \mathrm{IU} / 1$ & 10.2 & $5.3-13$ & 0.66 & & & \\
\hline & $<334$ IU/1 & 6.8 & $4.4-14.4$ & & & & \\
\hline$\gamma$-GTP & $\geq 66 \mathrm{IU} / 1$ & 6.8 & $4.5-13.6$ & 0.24 & & & \\
\hline & $<66 \mathrm{IU} / 1$ & 10.2 & $4.3-13.6$ & & & & \\
\hline Alb & $\geq 3.5 \mathrm{IU} / 1$ & 11.8 & $5.3-13.6$ & 0.56 & & & \\
\hline & $<3.5 \mathrm{IU} / 1$ & 5.8 & $3.0-13.6$ & & & & \\
\hline CRP & $\geq 1.0 \mathrm{mg} / \mathrm{dl}$ & 5.3 & $3.3-8.3$ & 0.085 & & & \\
\hline & $<1.0 \mathrm{mg} / \mathrm{dl}$ & 11.3 & $5.3-13.6$ & & & & \\
\hline CEA & $\geq 5.0 \mathrm{ng} / \mathrm{ml}$ & 6.1 & $4.4-13.6$ & 0.94 & & & \\
\hline & $<5.0 \mathrm{ng} / \mathrm{ml}$ & 11.2 & $4.5-14.4$ & & & & \\
\hline CA19-9 & $\geq 1,000 \mathrm{U} / \mathrm{ml}$ & 4.4 & $2.3-12.8$ & 0.009 & 1.72 & $0.77-3.86$ & 0.19 \\
\hline & $<1,000 \mathrm{U} / \mathrm{ml}$ & 11.3 & $5.3-15.7$ & & 1 & & \\
\hline BMI & $<22 \mathrm{~kg} / \mathrm{m}^{2}$ & 5.3 & $4.3-11.3$ & 0.20 & & & \\
\hline & $\geq 22 \mathrm{~kg} / \mathrm{m}^{2}$ & 12.2 & $5.3-14.4$ & & & & \\
\hline Sarcopenia & Present & 5.3 & $3.3-8.6$ & 0.045 & 2.50 & $1.15-5.43$ & 0.019 \\
\hline & Absent & 13.1 & $6.8-17.4$ & & 1 & & \\
\hline SATI & High & 12.9 & $6.3-16.4$ & 0.12 & & & \\
\hline & Low & 5.3 & $4.3-11.3$ & & & & \\
\hline VATI & High & 13.1 & $6.3-17.4$ & 0.023 & 2.05 & $0.83-5.10$ & 0.12 \\
\hline & Low & 5.3 & $4.3-10.1$ & & 1 & & \\
\hline ATI & High & 13.6 & $6.8-16.4$ & 0.027 & 1.20 & $0.33-2.13$ & 0.70 \\
\hline & Low & 5.3 & $4.5-11.3$ & & 1 & & \\
\hline
\end{tabular}

ALP: Alkaline phosphatase; ALT: alanine aminotransferase; AST: aspartate aminotransferase; ATI: adipose tissue index; BMI: body mass index; Bp: perihilar bile duct; CA19-9: carbohydrate antigen 19-9; CEA: carcinoembryonic antigen; CRP: C-reactive protein; CI: confidence intervaI; ECOG PS: Eastern Cooperative Oncology Group performance status; GEM: gemcitabine; HR: hazard ratio; SATI: subcutaneous adipose tissue index; T-Bil: total bilirubin; VATI: visceral adipose tissue index; WBC: white blood cell; $\gamma$ GTP: $\gamma$-glutamyl transpeptidase. Statistically significant $p$-values are shown in bold. 
A

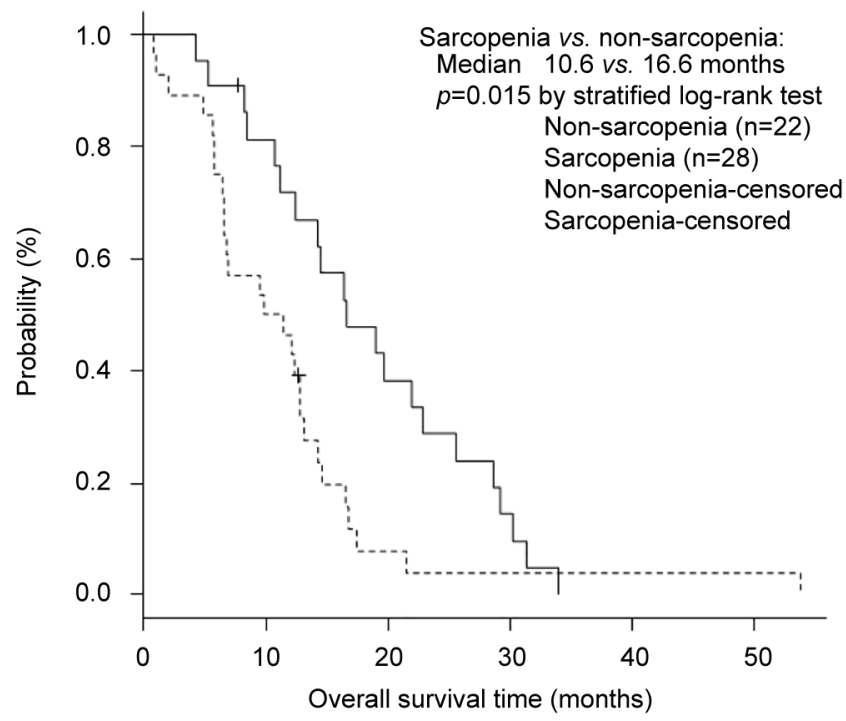

B

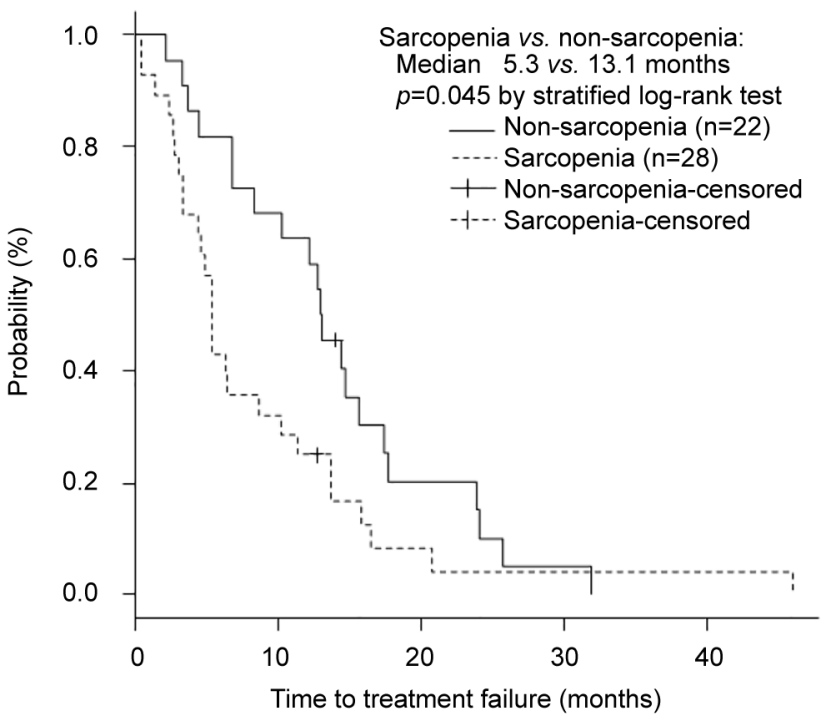

Figure 1. Kaplan-Meier curves for overall survival OS (A) and time to treatment failure (TTF) (B) in patients with and without sarcopenia.

loss of skeletal muscle mass was an independent predictor of poor prognosis in the postoperative period $(\mathrm{HR}=2.92$, 95\% CI=1.92-4.47; $p<0.001)(18)$.

The relationship between chemotherapy and sarcopenia has recently been reported for several types of carcinoma but reports on biliary tract cancer are limited. In their study of patients with advanced gastric cancer who underwent chemotherapy, Lee et al. showed that the OS was significantly shorter in patients with advanced gastric cancer with sarcopenia than those without (median: $6.8 \mathrm{vs} .10 .3$ months, $p=0.033$ ). Additionally, they revealed that sarcopenia was an independent prognostic factor for poor OS ( $\mathrm{HR}=1.51, p=0.029)$ (5). Kurita et al. studied the relation between sarcopenia and the effect of FOLFIRINOX in pancreatic cancer (9). They found that both OS and TTF were significantly shorter in the group with sarcopenia than in that without, and reported that sarcopenia was an independent prognostic factor for OS and TTF. Our findings on biliary cancer are similar to theirs for pancreatic cancer.

Recently, skeletal muscle has gained increasing attention as an endocrine organ involved in immune responses, including inflammatory reactions. Interleukin (IL) 15, which is highly expressed in the skeletal muscle, is required for the development and survival of natural killer lymphocytes. In patients with sarcopenia, a reduced expression of IL15 may lead to reduced innate immunity (19). Skeletal muscle cells also produce IL6, which is involved in the regulation of the immune system, and its role in the suppression of cancer cell growth has been described (20). Based on these findings, it is highly likely that patients with sarcopenia have a weakened immune system, which may have led to the present results.
This study has several limitations. This was a retrospective study with a relatively small sample size. Furthermore, there are no internationally standardized cut-off values for sarcopenia; here, we used the Japan Society of Hepatology criteria for sarcopenia. The Asian Working Group for Sarcopenia states that the cut-off value for sarcopenia varies according to race (4); however, standardized cut-off values for sarcopenia for each race have not been established. In addition, the revised European Working Group on Sarcopenia in Older People states that including data on low muscle strength/quality and low physical performance for the assessment of sarcopenia will help to better assess the severity and physical effects of sarcopenia, and this should be considered in future studies (21).

In conclusion, sarcopenia was identified as a factor for poor prognosis in patients with biliary tract cancer who underwent chemotherapy. Thus, maintenance of muscle mass may improve the efficacy of chemotherapy in such patients and consequently their prognosis.

\section{Conflicts of Interest}

All Authors declare no conflicts of interest.

\section{Authors' Contributions}

$\mathrm{KM}$ wrote the article. $\mathrm{KH}$ and ES participated in the critical revision of the article. KM, MS, YS, YT, YK, KK, and TS participated in the study as physicians who treated and followedup the patients with biliary tract cancer. $\mathrm{KM}, \mathrm{KH}$ and $\mathrm{YK}$ performed the statistical analyses. $\mathrm{KH}$ and $\mathrm{AN}$ were responsible for the design of the study. 


\section{Acknowledgements}

The Authors are grateful to ES for carefully proofreading the article. We would also like to thank Editage (htt://www.editage.jp) for English language editing.

\section{References}

1 Nagino M, Hirano S, Yoshitomi H, Aoki T, Uesaka K, Unno M, Ebata T, Konishi M, Sano K, Shimada K, Shimizu H, Higuchi R, Wakai T, Isayama H, Okusaka T, Tsuyuguchi T, Hirooka Y, Furuse J, Maguchi H, Suzuki K, Yamazaki H, Kijima H, Yanagisawa A, Yoshida M, Yokoyama Y, Mizuno T and Endo I: Clinical practice guidelines for the management of biliary tract cancers 2019: The $3^{\text {rd }}$ English edition. J Hepatobiliary Pancreat Sci 28(1): 26-54, 2021. PMID: 33259690. DOI: 10.1002/jhbp.870

2 Ishii H, Furuse J, Yonemoto N, Nagase M, Yoshino M and Sato T: Chemotherapy in the treatment of advanced gallbladder cancer. Oncology 66(2): 138-142, 2004. PMID: 15138366. DOI: $10.1159 / 000077440$

3 Rosenberg I: Summary comments. The American Journal of Clinical Nutrition 50(5): 1231-1233, 2018. DOI: 10.1093/ajcn/ 50.5.1231

4 Chen LK, Woo J, Assantachai P, Auyeung TW, Chou MY, Iijima K, Jang HC, Kang L, Kim M, Kim S, Kojima T, Kuzuya M, Lee JSW, Lee SY, Lee WJ, Lee Y, Liang CK, Lim JY, Lim WS, Peng LN, Sugimoto K, Tanaka T, Won CW, Yamada M, Zhang T, Akishita M and Arai H: Asian Working Group for Sarcopenia: 2019 consensus update on sarcopenia diagnosis and treatment. J Am Med Dir Assoc 21(3): 300-307.e2, 2020. PMID: 32033882. DOI: $10.1016 /$ j.jamda.2019.12.012

5 Buentzel J, Heinz J, Bleckmann A, Bauer C, Röver C, Bohnenberger H, Saha S, Hinterthaner M, Baraki H, Kutschka I and Emmert A: Sarcopenia as prognostic factor in lung cancer patients: a systematic review and meta-analysis. Anticancer Res 39(9): 46034612, 2019. PMID: 31519557. DOI: 10.21873/anticanres.13640

6 Lee JS, Kim YS, Kim EY and Jin W: Prognostic significance of CT-determined sarcopenia in patients with advanced gastric cancer. PLoS One 13(8): e0202700, 2018. PMID: 30125312. DOI: 10.1371 /journal.pone.0202700

7 Ishihara S, Horiguchi A, Miyakawa S, Endo I, Miyazaki M and Takada T: Biliary tract cancer registry in Japan from 2008 to 2013. J Hepatobiliary Pancreat Sci 23(3): 149-157, 2016. PMID: 26699688. DOI: $10.1002 /$ jhbp.314

8 Bertero L, Massa F, Metovic J, Zanetti R, Castellano I, Ricardi U, Papotti $\mathrm{M}$ and Cassoni P: Eighth Edition of the UICC Classification of Malignant Tumours: an overview of the changes in the pathological TNM classification criteria-What has changed and why? Virchows Arch 472(4): 519-531, 2018 PMID: 29209757. DOI: 10.1007/s00428-017-2276-y

9 Kurita Y, Kobayashi N, Tokuhisa M, Goto A, Kubota K, Endo I, Nakajima A and Ichikawa Y: Sarcopenia is a reliable prognostic factor in patients with advanced pancreatic cancer receiving FOLFIRINOX chemotherapy. Pancreatology 19(1): 127-135, 2019. PMID: 30473464. DOI: 10.1016/j.pan.2018.11.001

10 Haas M, Heinemann V, Kullmann F, Laubender RP, Klose C, Bruns CJ, Holdenrieder S, Modest DP, Schulz C and Boeck S: Prognostic value of CA 19-9, CEA, CRP, LDH and bilirubin levels in locally advanced and metastatic pancreatic cancer: results from a multicenter, pooled analysis of patients receiving palliative chemotherapy. J Cancer Res Clin Oncol 139(4): 681689, 2013. PMID: 23315099. DOI: 10.1007/s00432-012-1371-3

11 Tas F, Karabulut S, Ciftci R, Sen F, Sakar B, Disci R and Duranyildiz D: Serum levels of LDH, CEA, and CA19-9 have prognostic roles on survival in patients with metastatic pancreatic cancer receiving gemcitabine-based chemotherapy. Cancer Chemother Pharmacol 73(6): 1163-1171, 2014. PMID: 24647734. DOI: $10.1007 / \mathrm{s} 00280-014-2450-8$

12 Therasse P, Eisenhauer EA and Verweij J: RECIST revisited: a review of validation studies on tumour assessment. Eur J Cancer 42(8): 1031-1039, 2006. PMID: 16616487. DOI: 10.1016/j.ejca. 2006.01 .026

13 Kvist H, Sjöström L and Tylén U: Adipose tissue volume determinations in women by computed tomography: technical considerations. Int J Obes 10(1): 53-67, 1986. PMID: 3710689.

14 Hamaguchi Y, Kaido T, Okumura S, Kobayashi A, Hammad A, Tamai Y, Inagaki N and Uemoto S: Proposal for new diagnostic criteria for low skeletal muscle mass based on computed tomography imaging in Asian adults. Nutrition 32(11-12): 12001205, 2016. PMID: 27292773. DOI: 10.1016/j.nut.2016.04.003

15 Fujiwara N, Nakagawa H, Kudo Y, Tateishi R, Taguri M, Watadani T, Nakagomi R, Kondo M, Nakatsuka T, Minami T, Sato M, Uchino K, Enooku K, Kondo Y, Asaoka Y, Tanaka Y, Ohtomo K, Shiina S and Koike K: Sarcopenia, intramuscular fat deposition, and visceral adiposity independently predict the outcomes of hepatocellular carcinoma. J Hepatol 63(1): 131-140, 2015. PMID: 25724366. DOI: 10.1016/j.jhep.2015.02.031

16 Kanda Y: Investigation of the freely available easy-to-use software 'EZR' for medical statistics. Bone Marrow Transplant 48(3): 452-458, 2013. PMID: 23208313. DOI: 10.1038/bmt. 2012.244

17 Antoun S, Borget I and Lanoy E: Impact of sarcopenia on the prognosis and treatment toxicities in patients diagnosed with cancer. Curr Opin Support Palliat Care 7(4): 383-389, 2013. PMID: 24189893. DOI: 10.1097/SPC.0000000000000011

18 Okumura S, Kaido T, Hamaguchi Y, Fujimoto Y, Kobayashi A, Iida T, Yagi S, Taura K, Hatano E and Uemoto S: Impact of the preoperative quantity and quality of skeletal muscle on outcomes after resection of extrahepatic biliary malignancies. Surgery 159(3): 821-833, 2016. PMID: 26603849. DOI: 10.1016/j.surg.2015.08.047

19 Lutz CT and Quinn LS: Sarcopenia, obesity, and natural killer cell immune senescence in aging: altered cytokine levels as a common mechanism. Aging (Albany NY) 4(8): 535-546, 2012. PMID: 22935594. DOI: 10.18632/aging.100482

20 Karstoft $\mathrm{K}$ and Pedersen BK: Skeletal muscle as a gene regulatory endocrine organ. Curr Opin Clin Nutr Metab Care 19(4): 270-275, 2016. PMID: 27101470. DOI: 10.1097/ MCO.0000000000000283

21 Cruz-Jentoft AJ, Bahat G, Bauer J, Boirie Y, Bruyère O, Cederholm T, Cooper C, Landi F, Rolland Y, Sayer AA, Schneider SM, Sieber CC, Topinkova E, Vandewoude M, Visser M, Zamboni $\mathrm{M}$ and Writing Group for the European Working Group on Sarcopenia in Older People 2 (EWGSOP2), and the Extended Group for EWGSOP2: Sarcopenia: revised European consensus on definition and diagnosis. Age Ageing 48(1): 1631, 2019. PMID: 30312372. DOI: 10.1093/ageing/afy 169

Received May 26, 2021

Revised July 2, 2021

Accepted July 6, 2021 